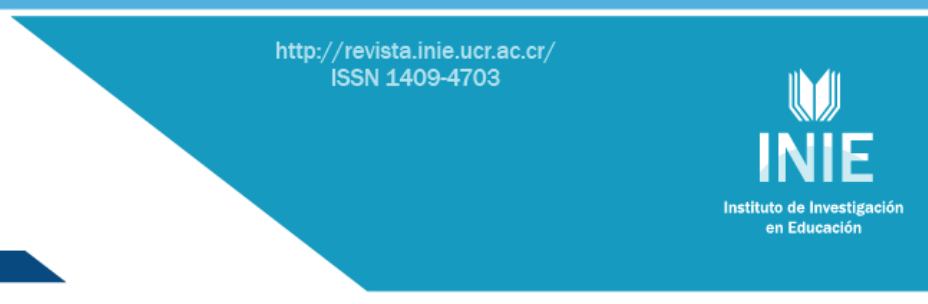

\title{
LAS HERRAMIENTAS MATEMÁTICAS EN LA FORMACIÓN TÉCNICO PROFESIONAL DEL TECNÓLOGO DE LA SALUD
}

THE MATHEMATICAL TOOLS IN THE TECHNICAL-PROFESSIONAL FORMATION OF THE HEALTH TECHNOLOGIST

\author{
Volumen 13, Número 3 \\ Setiembre - Diciembre
}

pp. $1-28$

Este número se publicó el 30 de setiembre de 2013

\author{
Juan R. Oliver Ventura \\ Jorge Manuel Ríos Obregón \\ Alejandro E. Estrabao Pérez \\ José Raúl Díaz López
}

Revista indizada en REDALYC, SCIELO

Revista distribuida en las bases de datos:

CATÁLOGO DE LATINDEX, IRESIE, CLASE, DIALNET, DOAJ, E-REVIST@S, SHERPA/ROMEO, QUALIS, MIAR

Revista registrada en los directorios:

ULRICH'S, REDIE, RINACE, OEI, MAESTROTECA, PREAL, CLASCO 


\title{
LAS HERRAMIENTAS MATEMÁTICAS EN LA FORMACIÓN TÉCNICO PROFESIONAL DEL TECNÓLOGO DE LA SALUD \\ THE MATHEMATICAL TOOLS IN THE TECHNICAL-PROFESSIONAL FORMATION OF THE HEALTH TECHNOLOGIST
}

\author{
Juan R. Oliver Ventura ${ }^{1}$ \\ Jorge Manuel Ríos Obregón² \\ Alejandro E. Estrabao Pérez ${ }^{3}$ \\ José Raúl Díaz López
}

Resumen: La propuesta didáctica que mostramos es parte del resultado del trabajo realizado en la Filial de Ciencias Médicas de Sancti Spíritus, Cuba. En él se propone un grupo de ideas para perfeccionar la formación Matemática del tecnólogo de la salud, el uso de herramientas Matemáticas en la resolución de problemas profesionales, su definición y significado dentro del proceso de enseñanza aprendizaje de esta ciencia. Se precisa la relación entre la formación de la cultura Matemática, la formación técnico profesional y la resolución de problemas de la profesión. Además, se hace referencia a la relación entre la visualización y la formalización Matemática como herramientas indispensables en el desarrollo del pensamiento tecnológico. El trabajo tiene el objetivo ejemplificar el uso de herramientas Matemáticas que facilitan el aprendizaje matemático con fines técnicos, la formación Matemática y la resolución de problemas profesionales. Esta aproximación nos permitió tratar con los fenómenos de producción y de difusión del conocimiento matemático desde la perspectiva del tecnólogo de la salud, al articular la epistemología del conocimiento, con su dimensión socio cultural, con los procesos cognitivos asociados a la práctica profesional con los mecanismos de institucionalización de la enseñanza.

Palabras clave: FORMALIZACIÓN, PROBLEMAS PROFESIONALES, HERRAMIENTAS MATEMÁTICAS, VISUALIZACIÓN, PENSAMIENTO TECNOLÓGICO, CUBA

\begin{abstract}
The following didactic proposal results from a work carried out at the Medical Sciences Branch from Sancti Spíritus, Cuba. It proposes a set of ideas to improve the mathematical training in health technologists, as well as the use of mathematical tools in solving professional problems, its definition and meaning within the learning process of this science. Throughout the work, the relationship between the mathematical culture education, the professional technical training and problem solving - regarding the profession - is clearly stated. It also refers to the relationship between mathematical visualization and formalization as essential tools in the technological thought development. This work aims to illustrate with examples the use of mathematical tools that facilitate mathematical learning for technical purposes, the mathematical training and professional problem solving. This approach allowed us to deal with the production and dissemination of mathematical knowledge phenomena from the health technologists' viewpoint, by putting the epistemology of knowledge with its sociocultural dimension together, as well as cognitive processes associated with professional practice including the institutionalization of education mechanisms.
\end{abstract}

Keywords: FORMALIZATION, PROFESSIONAL PROBLEMS, MATHEMATICAL TOOLS, VISUALIZATION, TECHNOLOGICAL THOUGHT, CUBA

\footnotetext{
${ }_{1}$ Profesor Asistente de la Universidad de Ciencias Médicas Dr. Faustino Pérez Hernández.

Sancti Spíritus, Cuba. Dirección electrónica: jventura@ssp.sld.cu

2 Profesor Auxiliar. Universidad José Martí. Sancti Spíritus, Cuba.

${ }^{3}$ Profesor Titular. Universidad de Oriente, Cuba.

${ }^{4}$ Profesor Titular. Universidad de Oriente, Cuba.
}

Artículo recibido: 3 de diciembre, 2012

Aprobado: 19 de agosto, 2013 


\section{INTRODUCCIÓN}

Los retos que asume hoy en día la formación del profesional universitario de las Ciencias Médicas deben ir en vías de potenciar su formación integral para contribuir con una sociedad que demanda la incorporación de profesionales competentes e investigadores. Esto es posible si se tiene en cuenta que dicha formación constituye un complejo proceso donde confluyen diversos componentes y subprocesos. Sin lugar a dudas, uno de los procesos que juega un rol importante en esta formación lo constituye el proceso de formación técnico profesional del estudiante universitario de las Ciencias Médicas.

El subsistema de Educación Médica incorpora los avances científicos y tecnológicos a la práctica médica, lo que demanda una actualización permanente de los contenidos que permitan la ejecución de los procesos tecnológicos con elevados niveles de conocimientos, habilidades y capacidades ante la fusión entre especialidades médicas y de estas con otras ramas del saber, por lo que son indispensables enfoques más generales e integrales en el proceso de formación de estos profesionales. Los problemas de la práctica científico técnica profesional del tecnólogo de la salud promueven las relaciones interdisciplinarias para su solución, lo que también refleja la complejidad de la propia realidad sobre la cual actúan.

Los tecnólogos de la salud, una vez que egresan de la universidad, desarrollan su actividad profesional en una pluralidad de niveles de atención, ya sea primario, secundario o terciario, y otras instituciones que coordinan las actividades del sector a nivel municipal y provincial, teniendo en cuenta complejas acciones preventivas, curativas y de rehabilitación en los servicios que brindan. Los esfuerzos metodológicos realizados y los avances obtenidos en la formación profesional dirigidos hacia el tratamiento de las funciones profesionales del tecnólogo de la salud apuntan a la existencia de dificultades en la ejecución de dichos procesos.

Al realizarse un diagnóstico en la Filial de Ciencias Médicas de Sancti Spíritus, Cuba, a través de la aplicación de diferentes instrumentos a 46 egresados de la carrera en las especialidades de Imaginología, Radiofísica Médica, Terapia Física y Rehabilitación, Servicios Farmacéuticos, Optometría y Óptica, Electromedicina, Higiene y Epidemiología y Administración y Economía, se reflejan manifestaciones externas en el modo de actuación y en el desempeño de este profesional, que se enmarcan en los escenarios docentes y asistenciales, entre otros, dados por: 
1. Imprecisiones en el proceso formativo de la Matemática dado por problemas de tipo metodológicos relacionados con el empleo de métodos y procedimientos que no activan el proceso y no prepara al estudiante para aprender por sí mismo en forma activa y permanente, fomentándole las capacidades para la obtención de información, la observación objetiva, el razonamiento lógico y el desarrollo del pensamiento tecnológico.

2. Limitado nivel de conocimientos de las ciencias que respaldan las diferentes tecnologías que le son pertinentes, lo que incide en cierta desvalorización de las potencialidades que le propician a su desempeño profesional diferentes disciplinas, que resultan objeto de tratamiento superficial y marginal.

3. Imprecisiones en la aplicación práctica de los conocimientos de la disciplina Matemática, donde no se ha propiciado suficientemente la integración interdisciplinaria y transdisciplinar.

4. Imprecisiones en el desarrollo del proceso tecnológico de la salud en el que interviene, debido a un nivel cognitivo insuficiente sobre la tecnología con la cual opera y el dominio del proceder, así como el poco intercambio y diálogo con el paciente.

En estos contextos de actuación, se observan limitaciones en la formación Matemática del tecnólogo de la salud que no favorecen su desempeño profesional, ante la demanda cada vez mayor de la atención especializada de la población, a partir de la existencia de un mayor conocimiento sobre la salud y la dinámica del desarrollo tecnológico donde está inmerso, generando nuevas exigencias en el desarrollo de su labor asistencial, docente, investigativa, gerencial y tecnológica. La comprensión por parte del profesional de la necesidad de perfeccionar cada vez más su modo de actuación para desempeñarse profesionalmente de una manera más eficiente es uno de los temas que necesitan un mayor tratamiento. La búsqueda de soluciones a los problemas de la práctica profesional en tecnología de la salud de manera contextualizada y el carácter interdisciplinario de los contenidos hacen necesario un enfoque integrador del proceso de formación de este profesional.

La asignatura Matemática, está dirigida para los estudiantes de $1^{\text {er. }}$ Año de las carreras de Tecnología de la Salud y tiene como precedente la asignatura Matemática Básica, aunque también algunos elementos de Álgebra Lineal, ambas tratadas en cursos anteriores. Matemática tiene como temas: Actualización de conocimientos. Teoría de Conjuntos, Números Complejos. Sistema de ecuaciones. Matrices y determinantes. Funciones, Límite y 
continuidad. Cálculo Diferencial de funciones reales de una variable real y el Cálculo Integral de funciones reales de una variable real. El Cálculo Integral abarca los temas de Integral Definida y sus aplicaciones, Integral Indefinida e Integrales impropias.

Una de las dificultades más significativas en la formación matemática de los alumnos de la Tecnología de la Salud, de la provincia Sancti Spiritus y del país, es su pobre preparación para enfrentar la resolución de ejercicios de aplicación, particularmente aquellos que constituyen problemas. La constatación realizada, a través de diferentes comprobaciones realizadas a los alumnos de la enseñanza media, ha servido para corroborar la dificultad señalada, detectándose las serias limitaciones para la búsqueda de una vía de solución de cualquier tipo de ejercicio y principalmente la pobre preparación para argumentar y llegar a conclusiones como resultado de la idea de la solución escogida, unido al hecho de que el desarrollo de las habilidades matemáticas no alcanzan el nivel de fijación y sistematización que satisfaga los objetivos de los programas de enseñanza.

El estudio de las dificultades detectadas ha promovido la necesidad de valorar la naturaleza de las causas de que tanto los profesores como los alumnos no alcancen resultados favorables en su nivel de formación para la resolución de problemas profesionales. El estudio de la Metodología de la enseñanza de la Matemática en lo que se refiere al tratamiento de los ejercicios, ha arrojado como conclusión: las grandes posibilidades para la estructuración del proceso de solución según las concepciones de Werner Jungk, Wolfgang Zillmer y George Polya, que son las más difundidas en el país; en ellas se describen de una manera muy práctica los procesos heurísticos que transcurren durante la búsqueda de la idea de la solución y la solución en sí, pero con la característica de que conciben el tratamiento de cada tipo de ejercicio, según el esquema heurístico propuesto.

Esto significa que el profesor prepara para cada tipo de ejercicio cómo proceder en el tratamiento de su solución, teniendo en cuenta si es un ejercicio formal o con texto, de demostración, de cálculo, construcción u otro. No obstante, la Metodología no se ocupa plenamente de cómo estructurar los diferentes niveles de organización de un curso (unidades temáticas, sistemas de clases y clases) que garantice un tratamiento sistemático y con una adecuada integración de los modos de actuación que deben asimilar los alumnos, es decir, estructurar los sistemas de clases, unidades temáticas y cursos a partir de la determinación de los modos de actuar, los métodos o estrategias de trabajo (habilidades más 
generales) y los procedimientos matemáticos en los que el profesor y los alumnos deben concentrar la mayor atención.

Teniendo en cuenta que escenario docente del aula no es suficiente para preparar el profesional de tecnología se diseñó las prácticas científico técnicas profesionales, como un ejercicio extra docente guiado y supervisado por el docente donde se ponen en juego los conocimientos adquiridos durante el proceso formativo del estudiante. Permiten concretizar teorías aplicándolas a situaciones problemáticas reales. Este ejercicio profesional posibilita a los estudiantes reconocer los límites de la teoría y acceder a los requerimientos de la realidad. Estas prácticas no tienen un solo sentido; establecen un diálogo continuo entre la formación recibida en la Universidad y la realidad.

La realidad en la que se insertan los estudiantes es un espacio que nutre los procesos de aprendizaje y contribuye a una comprensión más compleja y global de las problemáticas y situaciones del cuadro de salud del área en las que esté involucrado de acuerdo a la línea específica de la ubicación. Este ejercicio profesional propone un vínculo bidireccional en el que teoría y práctica se asimilan mutuamente, concretándose, dando lugar a un nuevo sentido y significado de la realidad social y profesional, constituye una experiencia multidimensional centrada en el aprender haciendo, entendido como aprendizaje en función de una interacción entre la experiencia y la competencia profesional.

\section{DESARROLLO}

La escuela constituye la institución que, de forma ineludible, tiene la tarea de preparar a niños y jóvenes para enfrentar la resolución de problemas como un objetivo instructivo y formativo, en el afán de alcanzar una formación integral para el desempeño en su vida laboral. El reconocimiento, por investigadores de diferentes tendencias y en diferentes sistemas educativos, de que la escuela no logra de forma óptima satisfacer tales exigencias, ocupa hoy el centro de interés en la mayoría de los eventos y foros internacionales en la discusión de la temática, lo que ha conducido al estudio y la búsqueda de alternativas para estructurar el proceso de enseñanza aprendizaje de tal forma que resolver problemas sea objeto de enseñanza y objeto de aprendizaje.

El aprendizaje de las Matemáticas es uno de los retos que enfrenta la didáctica contemporánea, la complejidad de este problema ha estado dado por distintos factores que se manifiestan en el proceso formativo del estudiante, existen varios elementos que 
complejizan el problema, entre ellos se puede citar la mala base de los estudiantes junto con la creencia errónea de que la Matemáticas es muy difícil de aprender y poco útil en alguna profesiones de las Ciencias Médicas, a esto se une a que los profesores ven la clase como una tarea a cumplir en un tiempo y un espacio dado, que no necesita de una preparación profunda, además la actitud del profesor en ocasiones es repetir el proceso de la misma forma como él aprendió, reproduciendo un proceso descontextualizado totalmente.

En tal sentido, el estudio de complejos de materia ayuda en la comprensión de las líneas directrices del programa, pero no concretan la labor de planificación y dirección que realiza el profesor en la escuela y que se corresponden con los documentos de la planificación (programas, libros de textos y orientaciones metodológicas) que precisan la información en estos niveles de organización. Un rasgo de la planificación y dosificación es que la resolución de problemas y de los ejercicios integradores del contenido estudiado aparecen, generalmente, al finalizar los sistemas de clases y unidades temáticas. De esta forma, el tiempo de que el alumno dispone para entrenarse en esta actividad de forma independiente tiende a ser mínimo, lo que conduce a pensar que no tiene suficiente oportunidad para su fijación y aplicación.

Una variación de esta concepción debe propiciar que la ejercitación, la profundización, sistematización y aplicación constituyan realmente momentos que propicien que la asimilación de los conocimientos y las habilidades matemáticas, se logre de forma integrada, desde el principio, a partir del objetivo a que se aspira (resolución de problemas) que permita formar en el alumno el modo de actuación frente a una determinada situación problemática. Para la resolución de ejercicios matemáticos hace falta un sistema de conocimientos activos, integrados, utilizables; sin embargo, la forma en que se planifican y dosifican los contenidos, según se señala, puede ser una causa de que no se logre una adecuada asimilación de su sistema de conocimientos y habilidades, teniendo en cuenta que una posición consciente hacia la solución de los ejercicios depende en alto grado de la capacidad del alumno para seleccionar y reestructurar los conocimientos y métodos necesarios.

El análisis tendrá en cuenta como cuestión central la permanencia estructurada de los conocimientos y las habilidades, desde el inicio, tomando como unidad organizativa la unidad temática; además de lograr que el alumno sea capaz de meditar y reflexionar 
alrededor de su propia actividad dirigida en lo fundamental hacia la resolución de problemas matemáticos.

Las tendencias pedagógicas que se manifiestan en la actualidad vinculadas al proceso docente educativo, destacan la importancia de la comunicación profesor - alumno y de la participación activa del estudiante. Esto impone nuevas exigencias al profesor, el cual debe generar un ambiente comunicativo en su grupo escolar, (...) más independiente y creadora y facilite una dinámica grupal positiva. (Vázquez, 2004, p.1)

La Matemática contemporánea ha adoptado ciertas metodologías de trabajo de las ciencias experimentales y son cada vez más importantes actividades como: observar, explorar, hacer predicciones, probar hipótesis, controlar variables, simular situaciones reales, sin dejar a un lado actividades como demostrar, generalizar o abstraer; sin que la Matemática sea considerada como una ciencia experimental los contenidos matemáticos han cambiado y también las formas de hacer matemáticas con el uso de equipos de cómputo y calculadoras, resaltando favorablemente la idea de que para el alumno se puede quedar a veces en lo experimental e intuitivo, no así el investigador y en la actualización de los profesores de la asignatura.

Aprender matemáticas ha dejado de ser comprendido como la simple acumulación de conceptos, teoremas o procedimientos en un determinado orden o relación, lo que ha conducido a que esta ciencia se comprenda como algo estático, como un complejo de términos y símbolos que el alumno tiene que dominar. El cuestionamiento de estas posiciones se hace partiendo de la idea de que aprender matemáticas es una actividad en la que el sujeto desarrolla o construye ideas, recopila información, descubre o crea relaciones, discute ideas, plantea conjeturas, valora resultados, pero que se realiza dentro de un contexto social que le aporta una significación que se va acercando al objeto. Aprender matemática es elaborar definiciones, más que repetir definiciones dadas por otros, es buscar ejemplos más que solicitarlos, es proponer contraejemplos cuando se quiere demostrar que una propiedad no es válida, es encontrar sentido a las hipótesis de un teorema, es hacerse preguntas además de responderlas.

Si a lo anterior se le agrega que en la actualidad el vertiginoso ritmo que alcanzan los avances científicos y técnicos obliga a la Didáctica a ponerse en correspondencia con ellos a escala mundial, la necesidad de dotar a los jóvenes egresados de las carreras de tecnología 
de la salud no solo de capacidades para asimilar y almacenar conocimientos, sino también estimular sus intereses por la búsqueda de métodos que le permitan apropiarse de estos conocimientos y se logre un mejor uso de sus habilidades profesionales, donde la Matemática juga un papel esencial. La necesidad de enseñar a aprender al alumno se hace cada vez más evidente, más racional, porque lograr que aprenda a aprender facilita el proceso de desempeño profesional en los contextos clínicos.

El proceso de enseñanza aprendizaje de las Matemáticas para los tecnólogos de la salud adquiere singular relevancia, ya que contribuye a elevar la calidad y esperanza de vida de la población, logrando un envejecimiento saludable y una longevidad satisfactoria. La preparación, formación y desarrollo de estos profesionales de la salud deben estar en correspondencia con los cambios tecnológicos modernos, ya que han de estar relacionados con el proceso de adquisición de equipos médicos de avanzada para elevar la calidad de los procesos clínicos, asistenciales, así como la prevención y curación de las enfermedades, todo lo cual se vincula con las necesidades que exigen transformaciones en las instituciones de salud. Es entonces una nueva posición o enfoque lo que se requiere en el proceso de formación de los tecnólogos de la salud.

El reconocimiento de la Educación Superior como proceso formativo trascendente, está determinado por el propósito de responder a los cambios del complejo mundo globalizado en que vivimos, y consecuente con la consideración de que constituye un espacio de apropiación social e intencional de la cultura universitaria, a través de las relaciones sociales de carácter formativo que se desarrollan entre los sujetos, como proceso de gestión cultural que conlleve a la transformación sustentable de la realidad. (FUENTES, 2009, p. 164)

En este sentido, la formación de la cultura Matemática del tecnólogo de la salud, requiere de la apropiación del conocimiento científico matemático prestándole especial importancia a su preparación, a los procesos del pensamiento matemático en el centro de la educación matemática, los impactos de la nueva tecnología y la conciencia de la importancia de la motivación. Como un proceso en el que es medular la relación entre la realidad y la matemática, la matemática vista como la ciencia que se encaminan a transmitir estrategias heurísticas adecuadas para la resolución de problemas más que a la transmisión de recetas ya elaboradas. A lograr la independencia cognoscitiva que debe caracterizarlos al egresar de 
la Universidad, sino por la complejidad tecnológica a la que se enfrentaran durante el ejercicio de su profesión y los complejos problemas profesionales que tendrán la posibilidad de darle solución. Y tanto la efectividad de la solución de estos como la realidad tecnológica en constante cambio hacen necesario el perfeccionamiento y desarrollo de su formación técnico profesional.

dentro del procesamiento de imágenes y abarca desde su adquisición hasta su procesamiento e interpretación como ayuda al diagnóstico. Aunque existen numerosos equipos de investigación dentro de las empresas que construyen los aparatos de adquisición, el análisis de estas imágenes como ayuda al diagnóstico es un campo en el que queda mucho camino por recorrer. Existen grupos de investigadores especializados en el estudio de imágenes de cada uno de los órganos del cuerpo humano: cerebro, corazón, hígado, sistema vascular, etc. En muchos de estos casos, el primer paso es la segmentación para la reconstrucción tridimensional del órgano en cuestión a partir de las imágenes dadas. Para mencionar un ejemplo que ilustra las múltiples implicaciones del problema, mencionemos el caso del estudio del sistema vascular: este estudio requiere la obtención de modelos anatómicos individualizados, el estudio y caracterización morfológica del dicho sistema o alguna de sus partes donde haya un problema, y eventuales simulaciones de dinámica de fluidos para comprender las presiones a que se ve sometida la estructura en cuestión. (Caselles, 2008, p. 1)

Lo más importante para esta investigación es que este autor introduce elementos como las creencias y las actitudes en las condiciones imprescindibles para la resolución de problemas y de los resultados de una amplia experiencia en el entrenamiento de los alumnos para resolver problemas profesionales e insiste en la necesidad de que el aprendizaje, en clases, se acerque lo más posible al modo de actuar del matemático, es decir, poder discutir ideas, negociar, especular sobre los posibles ejemplos y contraejemplos que ayuden a confirmar o a desaprobar sus ideas, lo que es muy importante tener en cuenta en la estructuración del proceso de enseñanza aprendizaje de la Matemática.

El problema profesional permite enfrentar nuevas vías de desarrollo, le permite visualizar situaciones concretas nuevas en etapas superiores del conocimiento personal. Contribuye, en gran medida, al desarrollo del pensamiento mediante la sistematización, generalización y la investigación de los problemas de la profesión. La situación dada en la 
profesión, proposición o dificultad que requiere solución, hecho o circunstancia que dificultan la consecución de algún fin en una profesión dada. Planteamiento de una situación cuya respuesta desconocida debe obtenerse.

El problema profesional es la configuración que caracteriza al proceso de enseñanza- aprendizaje en su vínculo con la necesidad social y por tanto, es la situación objetiva que se manifiesta en un proceso y se configura en el sujeto en la necesidad subjetiva de enfrentarlo, el cual constituye punto de partida del proceso. Es la situación que se da en un objeto y crea en el sujeto la necesidad de resolverla. Tiene un carácter objetivo al ser inherente al objeto, pero a la vez tiene un carácter subjetivo, determinado por el sujeto para quien la referida situación se convierte en necesidad.

El problema se transforma en el tiempo y espacio, en un contexto. Es multifacético, no se trata de un problema único y abstracto de por sí, sino realmente de un sistema de situaciones que en su abstracción sintetiza y concreta un nivel relacional cualitativamente superior, como configuración en construcción del conocimiento humano que responde a las necesidades humanas, por tanto el problema condiciona los objetivos formativos. El problema como categoría didáctica se delimita a los diferentes niveles de sistematicidad del proceso de enseñanza-aprendizaje, esto es, al nivel de la carrera, de las disciplinas docentes, las asignaturas y los temas.

Debido a la acumulación del conocimiento y los avances tecnológicos ocurridos en el sector de la salud, la asistencia médica ha cambiado profundamente en los últimos años. Ello se debe, en gran medida, al impresionante desarrollo de la tecnología médica. La aparición de algunas, imposibles de imaginar hace sólo unas décadas, han producido cambios muy significativos en la configuración de los servicios de salud. (Guerrero, 2004, p.6)

Es evidente que para lograr una formación Matemática del tecnólogo de la salud se requiere que este profesional se apropie de la cultura Matemática y de su dimensión profesional. La apropiación de la cultura Matemática es un proceso de intencionalidad formativa, que tiene su génesis en la actividad humana y se desarrolla en procesos de construcción de significados y sentidos, dinamizados en la contradicción entre la intencionalidad y la sistematización formativas, que se sustente en la relación dialéctica entre la naturaleza humana y su capacidad transformadora. Es una realidad del comportamiento 
de los profesionales en su desarrollo y se aprecia la formación Matemática en su desempeño profesional, son los conocimientos, habilidades, valores y valoraciones.

La apropiación de la cultura Matemática es síntesis, expresión y representación de la cultura general del individuo, en su entorno sociocultural y una posición epistemológica, a la vez que es dinámica como lo es la posición epistemológica y también la cultura del entorno, que en todo momento se trasforma en la propia apropiación de la misma por los sujetos, que a la vez renacen y se desarrollan. (Fuentes, 2009, p.175)

La formación técnico profesional del tecnólogo de la salud, al igual que su formación Matemática, como se ha dicho antes, al estar centrado en el desarrollo de la persona y ubicado en el contexto social parte de una concepción humanista. Al estar dirigida a la formación de un profesional integral con capacidad para transformar las necesidades y problemas que enfrenta en la comunidad en que se desempeña profesionalmente mediante un trabajo colectivo fundamentado en la integración de las funciones gerencial, asistencial, docente, investigativa y tecnológica, tiene una naturaleza holística.

La determinación del modelo del profesional a que se aspira, se formula en un lenguaje didáctico y responde a las necesidades sociales, que trasciende el marco de la institución universitaria y contiene las proyecciones que el país se plantea en un futuro de unos diez a quince años. De esta forma el modelo del profesional debe definir las características más esenciales del egresado, tanto en el ámbito profesional como social, que se pretende formar en las universidades, expresadas en sus aspectos no solo instructivos y educativos, sino de manera especial como nivel de síntesis en lo formativo, lo valorativo y lo ético, de ahí su carácter pedagógico. (Fuentes, 2009, p.10)

La formación de una cultura clínica donde el ambiente social, humano y la transformación de capacidades del ser humano mediante la formación del pensamiento donde se transforman las capacidades de los estudiantes y egresados de los tecnológicos de la salud de manera que tengan un desempeño profesional eficiente y eficaz, al estudiar esta problemática se enriquece con aportes provenientes de diversas disciplinas científicas y la Teoría Holístico Configuracional que aporta fundamentos sobre este proceso cultural y de crecimiento humano. 
La tecnología puede definirse como la aplicación del conocimiento científico a la solución de problemas prácticos y la obtención de metas humanas; un cuerpo de conocimientos desarrollados por una cultura que provee métodos o medios para controlar el entorno, extraer las fuentes, producir bienes y servicios, así como mejorar las condiciones de vida. El tecnólogo de la salud es un trabajador, tanto de la atención primaria como de la secundaria y la terciaria. Finalmente, puede decirse que, en nuestro entorno particular, se entiende por tecnología de la salud el conjunto de procederes empleados en la asistencia médica donde se aplica el conocimiento científico adquirido, con una estrategia, científicamente fundamentada y un enfoque clínico-epidemiológico-social y ecológico, con el objetivo de mejorar el estado de salud de la población, a partir de una tecnología sostenible, y con el anhelo de incrementar la calidad de vida de nuestro pueblo y crear un estado de bienestar pleno. (Guerrero, 2004, p.12)

El conjunto de teorías y de técnicas que permiten el aprovechamiento práctico del conocimiento científico, el conjunto de los términos técnicos, el lenguaje propio de una ciencia o de un arte determinado, al conjunto de los instrumentos y procedimientos industriales de un determinado sector o producto, es lo que asumimos como concepto de tecnología. En el sentido más amplio, la tecnología posibilita transformar el mundo, según las necesidades del hombre pueden relacionarse con aspiraciones humanas como el conocimiento, el arte o el control. La tecnología es un medio importante para crear entornos físicos y humanos nuevos. Sin embargo, los resultados de cambiar el mundo son impredecibles con frecuencia. Anticiparse a los efectos de la tecnología es tan importante como prever comprender sus potencialidades.

Ejemplo. Un farmacéutico debe preparar $15 \mathrm{ml}$ de unas gotas para los ojos para un paciente con glaucoma. La solución de las gotas debe contener $2 \%$ de un ingrediente activo, pero el farmacéutico sólo tiene una solución al 10\% y otra al 1\% en su almacén ¿Qué cantidad de cada tipo de solución debe usar para preparar la receta?

Solución. Sean $V_{i}$ : volumen de la i-ésima solución (en este caso $i=1,2$ ) y $C_{i}$ : concentración del i-ésimo ingrediente. 
Si queremos que la mezcla final tenga un volumen $V$ y una concentración $C$, debemos tener

$V C=V_{1} C_{1}+V_{2} C_{2} \quad$ y $\quad V=V_{1}+V_{2}$

Reemplazando los datos de nuestro problema, obtenemos

$15 \cdot 0.02=V_{1} 0.1+V_{2} 0.01$ y $15=V_{1}+V_{2}$

De esto, $0.3=0.1 V_{1}+0.01\left(15-V_{1}\right)$, y despejando obtenemos el valor $V_{1}=1.667 \mathrm{ml}$ y

$V_{2}=13.333$. Luego, para preparar $15 \mathrm{ml}$ de gotas al $2 \%$ debemos utilizar $1.667 \mathrm{ml}$ de solucion al $10 \%$ y $13.333 \mathrm{ml}$ de solucion al $1 \%$.

El aprendizaje matemático contemporáneo está sustentado en la resolución de problemas, el uso de las nuevas tecnologías se ha generalizado en el proceso didáctico de esta ciencia. La computadora como herramienta mediadora e instrumental en los procesos de formación constituye un innegable recurso didáctico que al incorporarse como auxiliar al desempeño de los profesionales le aporta efectividad, agilidad y humaniza el desempeño de los tecnólogos de la salud. La resolución de problemas prácticos de la vida cotidiana ha sido un factor decisivo para el desarrollo del hombre. La capacidad de razonar, única en su especie, le ha permitido transformarse y transformar el mundo en que vive para satisfacer sus propias necesidades.

El carácter y la naturaleza de las herramientas Matemáticas es algo vital dentro del proceso de desarrollo de capacidades cognitivas. Hacer Matemática en los contextos de desempeño profesional es vital en los tiempos eminentemente tecnológicos desde una perspectiva más amplia que la acotada en los textos matemáticos modernos. El uso del aprendizaje por proyectos junto con las diferentes acciones que se desarrollan en la construcción de un pensamiento matemático dotado de recursos técnicos matemáticos que se pueden llamar herramientas Matemáticas permite al joven profesional desarrollar habilidades para la resolución de problemas de la profesión.

El desarrollo del pensamiento en la Matemática o las contribuciones que hace la Matemática a su desarrollo tiene una relación directa con la formalización del conocimiento de la Matemática escolar y tecnológica porque constituye un proceso abstracto, cíclico y 
progresivo propio del proceso formativo que permite la construcción del conocimiento matemático sobre la base de axiomas simbólico-verbales y nuevas propiedades deducidas por procesos formales; expresando la continuidad lógica de los métodos de esta disciplina Matemática en la realización y ejecución y en la propia construcción del conocimiento matemático a partir de la apropiación de la cultura de esta ciencia.

El proceso formativo tiene varias categorías esenciales que desarrollan este proceso y le garantizan su carácter de continuidad y consecutividad a niveles superiores en la construcción científica del contenido socio cultural por el sujeto, donde se significan factores y criterios que propician la reestructuración epistemológica de ese contenido y con ello su sistematización, lo que condiciona la profundización del contenido en los sujetos. La sistematización, por tanto, es una categoría pedagógica y didáctica, que conlleva a la recreación y creación de la cultura, al revelar relaciones estructurales que propician nuevas relaciones de síntesis y de la estructura epistemológica y praxiológica del contenido, donde desempeña un rol fundamental en los primeros estadios del proceso de la construcción científica del contenido socio cultural que se construye.

La sistematización de la cultura y la experiencia en desarrollo, vista en lo perenne, la secuencialidad, la persistencia y la prolongación de otros subprocesos, propicia la reorientación del quehacer formativo e investigativo, en los procesos en desarrollo humano, así como también el rescate de experiencias y conocimientos históricamente construidos. Significa que la sistematización tiene un condicionamiento histórico, social y cultural en un contexto concreto, que condiciona el desarrollo ulterior de la cultura, la formación y la investigación. Los criterios de sistematización formativa emergen del propio proceso de la sistematización.

La sistematización como proceso inherente al desarrollo humano es considerada como categoría y dinámica del movimiento, por tanto, sienta las pautas de los procedimientos a seguir en el proceso formativo, lo que permite dar continuidad y consecutividad al mismo desde la perspectiva de un sistema de vías, alternativas; en tal sentido emerge de la contradicción entre la apropiación de la cultura y la profundidad del contenido. La apropiación de la cultura es un proceso humano que no puede ser reducido a un proceso cognitivo, sino a una ámbito de mayor trascendencia, que comprenda a la práctica humana, la valoración y la comunicación, de ahí que la educación superior sea un proceso de construcción científica de la capacidad transformadora humana. 
El resultado de los procesos integrados entre el proceso de enseñanza-aprendizaje de la Matemática, el uso de herramientas Matemáticas y la resolución de problemas profesionales, donde medien las tecnologías vinculadas a la profesión, contribuyen a la formación del pensamiento tecnológico del profesional. La Matemática, como una de las ciencias más antiguas, está presente a lo largo del desarrollo de la humanidad. Los conocimientos matemáticos fueron adquiridos por los hombres desde las primeras etapas del desarrollo bajo la influencia de la actividad productiva. A medida que se desarrollaban las formas de producción la actividad cambió y creció el conjunto de factores que influían en el desarrollo de las Matemáticas.

Desde que el hombre fue capaz de manifestarse existen evidencias de rasgos matemáticos en su legado histórico, en los diseños prehistóricos de cerámica, tejidos y en las pinturas rupestres se pueden encontrar evidencias del sentido geométrico y del interés en figuras geométricas. Los sistemas de cálculo primitivos estaban basados, seguramente, en el uso de los dedos de una o dos manos, lo que resulta evidente por la gran abundancia de sistemas numéricos en los que las bases son los números 5 y 10 .

Desde su surgimiento la Matemática tuvo dentro de su objeto el de darle solución a problemas concretos de las Ciencias Naturales y a la formación de conceptos. La aparición de las Matemáticas en las Ciencias Naturales fue el resultado de la aplicación de las teorías Matemáticas existentes a problemas prácticos y de la elaboración de nuevos métodos para su resolución.

El matemático y filósofo Pitágoras, con su escuela de pensamiento donde las Matemáticas se convirtieron en disciplina fundamental en toda investigación científica. En la Academia de Platón se establecía el razonamiento deductivo y las representaciones Matemáticas. En el Liceo de Aristóteles primaban el razonamiento inductivo y la descripción cualitativa. La interacción entre estos dos enfoques de la ciencia ha llevado a la mayoría de los avances posteriores. Euclides, Arquímedes y Apolonio, geómetras Griegos, que crearon un precedente geometría en Grecia que no pudo ser igualado por ningún otro griego.

En 1821, un matemático francés, Augustin Louis Cauchy, consiguió un enfoque lógico y apropiado del cálculo. Cauchy basó su visión del cálculo sólo en cantidades finitas y el concepto de límite. El matemático alemán Julius W. R. Dedekind encontró una definición adecuada para los números reales. El también matemático alemán Peter G. L. Dirichlet logró definir el significado de la palabra función, quien propuso su definición en los términos 
actuales. El matemático británico George Boole describió en Investigación sobre las leyes del pensamiento (1854) un sistema algebraico que se conoció más tarde como álgebra de Boole. Este sistema tiene numerosas aplicaciones prácticas en informática.

A principios del siglo, Carl Friedrich Gauss dio una explicación adecuada del concepto de número complejo; estos números formaron un nuevo y completo campo del análisis. Otro importante avance del este campo fue el estudio, por parte de Fourier, de las sumas infinitas de expresiones con funciones trigonométricas. Las que se conocen hoy como series de Fourier, y son herramientas muy útiles tanto en las Matemáticas puras como en las aplicadas. La teoría de Cantor, que fue considerada como demasiado abstracta y criticada como "enfermedad", forma hoy parte de los fundamentos de las Matemáticas y recientemente ha encontrado una nueva aplicación en el estudio de corrientes turbulentas en fluidos.

El conocimiento matemático del mundo moderno está avanzando más rápido que nunca. Teorías que eran completamente distintas se han reunido para formar teorías más completas y abstractas. Aunque muchos de los problemas más importantes han sido resueltos, otros como las hipótesis de Riemann siguen sin solución. Al mismo tiempo, siguen apareciendo nuevos y estimulantes problemas. Incluso las Matemáticas más abstractas están encontrando aplicación.

\section{Las herramientas Matemáticas para la resolución de problemas profesionales}

Muchos de los investigadores que han dedicado su trabajo a la resolución de problemas parten en sus argumentaciones que es un caso especial de aprendizaje significativo. Los cambios tecnológicos y el desarrollo acelerado de las ciencias exigen que los estudiantes tengan las herramientas para enfrentar la profesión y la Matemática debe darle las vías para entender y enfrentar la vida profesional con éxito. Esta idea viene acompañada por una creciente aceptación del punto de vista constructivista de las cogniciones, de donde sigue un serio examen sobre la evidencia de que, en esas situaciones, las cogniciones están situadas y distribuidas, antes que puedan ser consideradas en forma descontextualizada o como productos exclusivos de mentes individuales.

Ejemplo: para medir la capacidad respiratoria de los pulmones, se hace una prueba que consiste en inspirar al máximo y después espirar tan rápido como se pueda en un 
aparato llamado "espirómetro". Esto se puede expresar a través de una curva que indica el volumen de aire que entra y sale de los pulmones.

Muchas serían las preguntas que pudieran formular relacionada con esta situación y la gráfica dada. Un tecnólogo de la salud debe tener las herramientas para resolver situaciones como esta ¿cuál es el volumen en el momento inicial?, ¿cuánto tiempo duró la observación?, ¿cuál es la capacidad máxima de los pulmones de esta persona?, ¿cuál es el volumen a los 10 segundos de iniciarse la prueba? y otras.

Una herramienta Matemática no es necesariamente un objeto elaborado para hacer una actividad determinada, es mucho más, es un recurso mental que permite articular el pensamiento con la acción profesional, es un proceso dentro de la propia Matemática, que necesita saber un estudiante universitario o un profesional para enfrentar situaciones como esta. Poseer los recursos matemáticos o herramientas que me permitan resolver problemas de la vida o de la profesión es algo con lo que sueñan muchos estudiantes y profesionales. No es necesario ser matemático o un estudioso de esta ciencia para poseer herramientas importantes para enfrentar la vida.

\section{¿A que llamamos herramientas Matemáticas?}

Son una técnica del conocimiento, capacidades para el uso de recursos matemáticos, destrezas para resolver problemas, uso e interpretación de tecnologías, instrumentos matemáticos, uso de herramientas profesionales y tecnológicas, recursos humanos y materiales; materias primas, productos obtenidos, trabajo con desechos y residuos. Cuestiones relacionadas con la actividad profesional productiva, la distribución de productos, usuarios y consumidores, entre otras. En resumen, las herramientas Matemáticas son procesos simples de las Matemáticas que facilitan la comprensión y resolución de problemas simples, como ejemplos: la aplicación de la regla de tres simple, la descomposición de factores, la simplificación reducción o ampliación de fracciones algebraicas, el cálculo de límites elementales, el cálculo de derivadas sencillas, escribir la ecuación de una resta, o una curva y otras. Las herramientas de que hablamos son procesos sencillos de la Matemática, pero completos, por sí solos expresan algo o dan una solución a un problema sencillo. La herramienta Matemática es un conocimiento útil y perdurable.

Algunos de los conocimientos previos que necesitan los estudiantes para el trabajo con funciones, son herramientas necesarias para su trabajo dentro de su desempeño profesional. 


\section{Ejemplo 1}

Se pretende instalar una planta de ósmosis inversa destinada a la producción de agua potable. La planta captará aguas subterráneas con una concentración media de nitratos de $40 \mathrm{mg} \mathrm{N} / \mathrm{L}$. El agua producida tendrá una concentración de nitratos de $3 \mathrm{mg} \mathrm{N} / \mathrm{L}$. El agua de rechazo de la planta (un $40 \%$ del agua subterránea captada) será evacuada a una laguna litoral. La administración ha fijado como objetivo de calidad de nitratos en la laguna un valor de $2 \mathrm{mg} \mathrm{N} / \mathrm{L}$. Calculad:

- El caudal máximo de agua potable que puede producir la planta para que no se sobrepase en la laguna el objetivo de calidad.

- El tiempo que tardará en alcanzarse el objetivo de calidad una vez iniciada la actividad. Se asume que, en el periodo de tiempo considerado, los nitratos son contaminantes conservativos.

Características de la laguna: Caudal natural de entrada $Q_{e}=5 \mathrm{~m}^{3} / \mathrm{s}$; Caudal natural de salida $Q_{s}=Q_{e}$; Concentración de nitratos en el caudal de entrada $\left[\mathrm{NO}_{3}\right]_{e}=1 \mathrm{mg} \mathrm{N} / \mathrm{L}$; Concentración inicial de nitratos en la laguna (antes del inicio del vertido) $\left[\mathrm{NO}_{3}^{-}\right]_{0}=1 \mathrm{mg} \mathrm{N} / \mathrm{L}$; Superficie: $10^{5} \mathrm{~m}^{2}$; Profundidad media: $2 \mathrm{~m}$.

Solución: En primer lugar hay que determinar la concentración de nitratos en el agua de rechazo. Sea $Q_{c}$ el caudal de agua subterránea captado, $Q_{p}$ el caudal de agua potable producido, y $\mathrm{Q}_{\mathrm{r}}$ el caudal de rechazo. Aplicando un balance de materia para la producción de agua potable:

$$
\begin{aligned}
& Q_{c}\left[\mathrm{NO}_{\overline{3}}^{-}\right]_{c}=Q_{p}\left[\mathrm{NO}_{\overline{3}}^{-}\right]_{p}+Q_{r}\left[\mathrm{NO}_{\overline{3}}^{-}\right]_{r} \\
& Q_{c} 40=0.6 Q_{c}\left[\mathrm{NO}_{\overline{3}}^{-}\right]_{p}+0.4 Q_{c}\left[\mathrm{NO}_{\overline{3}}^{-}\right]_{r} \\
& 40=0.63+0.4\left[\mathrm{NO}^{-}\right]_{r} \\
& {\left[\mathrm{NO}_{\overline{3}}^{-}\right]_{r}=95.5 \mathrm{mg} \mathrm{N} / \mathrm{L}}
\end{aligned}
$$

Para calcular el caudal máximo de agua potable que se puede producir, se aplica un balance de materia al lago con el vertido del rechazo en estado estacionario:

$$
\begin{aligned}
& V \frac{d\left[N O_{\overline{3}}^{-}\right]}{d t}=Q_{e}\left[N O_{\overline{3}}^{-}\right]_{e}+Q_{r}\left[N O_{\overline{3}}^{-}\right]_{r}-\left(Q_{e}+Q_{r}\right)\left[N O_{3}^{-}\right]=0 \\
& 0=5 \mathrm{~m}^{3} / \mathrm{s} 1 \mathrm{mgN} / \mathrm{L}+Q_{r} 95.5 \mathrm{mgN} / \mathrm{L}-\left(5+Q_{r}\right) 2 \mathrm{mgN} / \mathrm{L} \\
& 0=5+Q_{r} 95.5-10-Q_{r} 2 \\
& Q_{r}=0.053 \mathrm{~m}^{3} / \mathrm{s}
\end{aligned}
$$


de donde: $\left\{\begin{array}{l}Q_{c}=\frac{Q_{r}}{0.4}=\frac{0.053}{0.4}=0.1325 \mathrm{~m}^{3} / \mathrm{s} \\ Q_{p}=0.6 Q_{c}=0.0795 \mathrm{~m}^{3} / \mathrm{s}\end{array}\right.$

Para calcular el tiempo que tardará la laguna en alcanzar el objetivo de calidad fijado, se parte igualmente de la ecuación del balance de nitratos:

$V \frac{d\left[\mathrm{NO}_{3}^{-}\right]}{d t}=Q_{e}\left[\mathrm{NO}_{3}^{-}\right]_{e}+Q_{r}\left[\mathrm{NO}_{3}^{-}\right]_{r}-\left(Q_{e}+Q_{r}\right)\left[\mathrm{NO}_{3}^{-}\right]$

Llamando $\left\{\begin{array}{l}W=Q_{e}\left[N O_{3}^{-}\right]_{e}+Q_{r}\left[N O_{3}^{-}\right]_{r}=51+0.05395 .5=10.273 \mathrm{~g} / \mathrm{s} \\ B=\frac{Q_{e}+Q_{r}}{V}=\frac{5+.053}{200000}=2.52610^{-5} \mathrm{~s}^{-1}\end{array}\right.$

Cuya integración resulta:

$\left[N O_{3}^{-}\right]=\frac{W}{B V}\left(1-e^{-B t}\right)+\left[N O_{3}^{-}\right]_{0} e^{-B t}$

$2=\frac{10.273}{2.52610^{-5} 200000}\left(1-e^{-2.52610^{-5} t}\right)+1 e^{-2.52610^{-5} t}\{$

$2=2.033-2.033 e^{-2.56610^{-5} t}+e^{-2.56610^{-5} t}$

$1.033 e^{-2.52610^{-5} t}=0.033$

$t=136331 s=37.87$ horas

El análisis de las estructuras internas del cuerpo solo ha sido posible durante el último siglo, con el descubrimiento de los rayos $X$. Otra revolución llegó no hace más de 30 años con la utilización de las tomografías computarizadas y resonancias magnéticas que permiten el estudio y análisis en tres dimensiones del cuerpo humano. Es así como el campo de la imagenología médica se convierte en una de las aplicaciones más importantes del procesamiento y visualización de imágenes en realidad virtual. Este campo de la medicina, cuyo desarrollo empezó a principios del siglo XX con el descubrimiento de los rayos $\mathrm{X}$ por Wilhelm Röntgen y que hasta hace unos cuantos años se basaba exclusivamente en la lectura de imágenes en 2D por parte de los especialistas, se encuentra en una constante evolución hacia el desarrollo de tecnologías que permitan también realizar reconstrucciones 
tridimensionales teniendo las mismas bases de los equipos convencionales de Ultrasonido (ecografía), Tomografìa Axial Computarizada (CT), resonancia magnética (RM) y Tomografía por emisión de positrones (PET) que con la ayuda de gran cantidad de software y hardware capaz de tomar estas imágenes para convertirlas en representaciones 3D bastante cercanas a la realidad que ya pueden ser trabajadas como imágenes digitalizadas en la red.

La enseñanza de la Matemática es un proceso al cual lo limitan varios factores como: la motivación, la solidez de los conocimientos de la Matemática elemental que se deben garantizar en la enseñanza precedente, (a los que también llamamos conocimientos previos o precedentes), la orientación hacia la profesión, la objetividad de los programas de Matemática escolar, los métodos de enseñanza y aprendizaje que intervienen en el proceso formativo, tanto en docencia como en el desempeño profesional.

La relación entre métodos heurísticos generales y la importancia del contenido específico ha sido un tema de controversia cuando se aborda la discusión del desarrollo de la inteligencia. El dilema puede tomar diversas formas: Si una idea o heurística aprendida es demasiado específica, entonces no se puede esperar una transferencia fácil a otras situaciones. Por otro lado si la idea se presenta en forma general no parece claro cuándo el dominio de esa idea realmente se ha logrado." (Santos Trigo, 1994, p.15)

La formación Matemática del tecnólogo de la salud gestiona e influye en la formación general del estudiante, como proceso parte de un diagnostico intencional, determina toda la necesidad y carencias cognitivas de los estudiantes proponiendo etapas para su solución parte del uso de recursos matemáticos que tienen mucho que ver con las etapas para la resolución de problemas definida por Polya (Análisis del Problema, Trazar un plan para resolverlo, Poner en práctica el plan y Comprobar el resultado).

La resolución de problemas profesionales es un proceso donde se integran las cuatro fases generales de la resolución de problemas y sus principales invariantes. Comienza con la clasificación o identificación del tipo de problema y la determinación de las herramientas Matemáticas necesarias, inicialmente se establece un algoritmo para la resolución pero deben lograrse procesos mentales que den respuesta en cada momento. En este proceso se sigue el procedimiento general para la solución de un problema cualquiera. Durante este proceso es necesario interactuar con herramientas Matemáticas que aportan los recursos 
necesarios para enfrentar problemas profesionales desde los elementales hasta los más complejos. Entiéndase como herramientas al conjunto de procesos parciales de las Matemáticas, instrumentos, artificios para resolver problemas. Procesos o parte de procesos matemáticos que permiten llegar a resultados intermedios dentro de un problema de la profesión. Por ejemplo: descomponer en factores, plantear una ecuación o sistema, calcular porcientos, elaborar gráficos, racionalizar denominadores y otros.

\section{¿Se pueden considerar herramientas Matemáticas a la visualización y la formalización?}

La visualización en Matemáticas son la representación de las ideas, conceptos y métodos matemáticos que enriquecen los contenidos matemáticos desde el punto de vista visual, facilita la representación gráfica, geométrica, numérica o algebraica, cuya utilización resulta provechosa para la solución de problemas, como para expresar algo Matemáticamente o en la práctica cotidiana. La visualización es por sí sola una herramienta que permite expresar visualmente ideas, modos de precesión de conceptos y métodos de solución de problemas o ejercicios donde la Matemática juega un papel importante para su solución.

La visualización se convierte en un aspecto extraordinariamente importante dentro de la Matemática, es natural, es algo que se convierte en una parte esencial del trabajo matemático, es un complemento de las abstracciones Matemáticas. Es un proceso sumamente útil a la hora de comprender las relaciones de la vida, de establecer las estructuras comunes de las cosas y de producir efectos en las cosas cuando sea oportuno. Cuando parece que las actividades Matemáticas de la abstracción nos alejan de la realidad visible muy a menudo el simbolismo matemático, diagramas y otros recursos de la imaginación nos facilita acercarnos al objeto de estudio y proponer un conjunto de alternativas para percibir la solución al estudio determinado. La visualización es algo natural dentro del pensamiento matemático, el descubrimiento de nuevas relaciones entre los objetos matemáticos, y también en comunicación de ideas propias de la Matemática.

El formalismo matemático tiene como importancia la construcción de los conocimientos matemáticos, no es el resultado en sí, sino la ley que establece y estructura las relaciones entre los objetos matemáticos. Establece las relaciones lógico-Matemáticas, que dan como resultado la estructura Matemática. El formalismo matemático se forma en una idea de objeto 
en cuestión que luego se sustenta en leyes, reglas y postulados que establecen el objeto como tal, diferenciándolo de otros objetos. En resumen, la formalización es el establecimiento de leyes o reglas que definen los objetos en sí.

Ejemplo: en la formalización Matemática del concepto de límite se utilizan varias concepciones, entre ellas la topológica, que parte de axiomas simbólicos-verbales como los de cantidades infinitamente grande y cantidades infinitamente pequeñas (infinitesimales) que unidas al concepto de distancia métrica, definido a partir tres axiomas, permiten la construcción de los concepto de vecindad de un punto y el de punto de acumulación que hacen posible formalizar la noción de que el límite de una función $y=f(x)$ cuando la variable independiente se aproxima (o tiende) a un punto $x_{o}$, es el número $L$, al que se aproxima la variable dependiente y, escrito de la forma: $f(x)=L i$.

\section{Ejemplos del uso de herramientas Matemáticas en la formación del tecnólogo}

\section{de la salud.}

1. Ejemplo: Calcule los límites de las funciones en los puntos indicados.

$$
\begin{gathered}
\lim _{x \rightarrow-1}(3 x+7)=3(-1)+7=4(3 x+7)=3(-1)+7=4 \\
\lim _{x \rightarrow-1} \frac{2 x^{2}-3 x+8}{5 x^{3}-7}=\frac{2(-1)^{2}-3(-1)+8}{5(-1)^{3}-7}=-\frac{13}{12} \\
\lim _{x \rightarrow 8} \frac{x^{2 / 3}+3 \sqrt{x}}{4-(16 / x)}=\frac{\lim _{x \rightarrow 8} x^{2 / 3}+3 \lim _{x \rightarrow 8} \sqrt{x}}{\lim _{x \rightarrow 8} 4-\lim _{x \rightarrow 8}(16 / x)}=\frac{8^{2 / 3}+3 \sqrt{8}}{4-(16 / 8)}=\frac{4+6 \sqrt{2}}{2}=2+3 \sqrt{2}
\end{gathered}
$$

2. Represente gráficamente las siguientes funciones elementales: (Ver cuadro) 


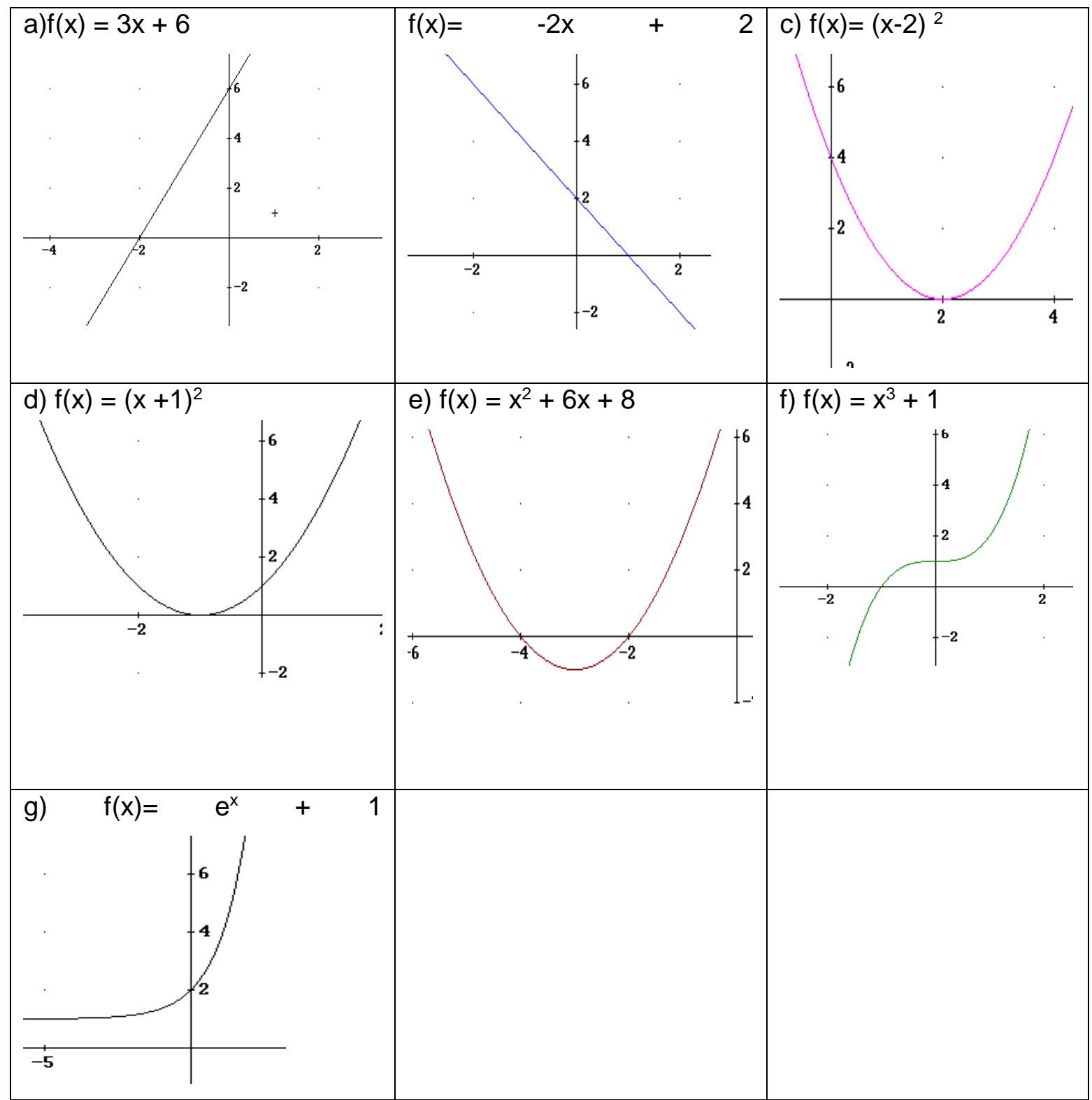

3. La concentración de cierto calmante suministrado mediante suero varía en su efectividad en el tiempo según la expresión $C=t^{2}-2 t+5$, donde $C$ se mide en miligramos por litros y el tiempo t en horas. Se determinó que el calmante no produce daños colaterales y es efectivo si la concentración es de por lo menos 8 miligramos por litros y a lo más 13 miligramos por litros ¿Durante cuánto tiempo es efectivo el calmante? 
Solución. De acuerdo con los datos aportados por el planteo del problema, para tener efectividad del calmante $8 \leq \mathrm{t}^{2}-2 \mathrm{t}+5 \leq 13$. Entonces es necesario resolver las inecuaciones: $8 \leq \mathrm{t}^{2}-2 \mathrm{t}+5 \mathrm{y} \mathrm{t}^{2}-2 \mathrm{t}+5 \leq 13$

Resolviendo la inecuación $8 \leq \mathrm{t}^{2}-2 \mathrm{t}+5$, comparando con cero, o restando 8 en ambos miembros de la desigualdad, se tiene. $0 \leq t^{2}-2 t-3$, entonces se obtiene que: $0 \leq(t+1)(t-3)$, luego obtenemos:

$0 \leq(\mathrm{t}+1)(\mathrm{t}-3)$ si $\mathrm{t} \epsilon]-\infty,-1] \cup[3,+\infty[$

Luego, buscamos los que satisfacen la desigualdad: $t^{2}-2 t+5 \leq 13$, de donde $(t-4)(t+2) \leq 0$, haciendo el corrimiento de signos, obtenemos que $(t-4)(t+2) \leq 0$ si $t \in[-2,4]$, interceptando las soluciones anteriores tenemos que el calmante será efectivo entre 3 y 4 horas después de administrado.

4. Un paciente con cáncer recibirá terapia mediante fármacos y radiación. Cada centímetro cúbico de medicamento que se usará contiene 200 unidades curativas, y cada minuto de exposición a la radiación proporciona 300 unidades curativas. El paciente requiere 2,400 unidades curativas. Si d centímetros cúbicos de la droga y $r$ minutos de radiación son administrados, determine la función lineal que relaciona $d$ y $r$. Grafique e interprete los resultados.

Solución. De los datos aportados en el problema se tiene que $200 d+300 r=2400$. A partir de esta igualdad, expresamos $d$ como función lineal de $r$ por $d(r)=12-3 r$. El gráfico de la función d es:

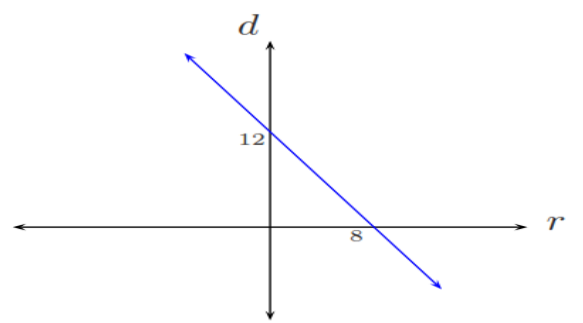

De la figura se desprenden algunos datos interesantes. Por ejemplo, si no se usara radiación en el tratamiento, se deben administrar al paciente $12 \mathrm{~cm}^{3}$ de droga. Por otra parte, si no se usan drogas en el tratamiento, son necesarios 8 minutos de radiación.

Observe que la función es decreciente, esto nos indica que mientras más minutos de radiación se apliquen al paciente, se administrará menos droga. El problema tiene sentido para $r$ entre 0 y 8. 
5. Un fabricante ha desarrollado un nuevo diseño de paneles de energía solar. Los estudios de mercado indican que la demanda anual de paneles depende del precio que se cobre por ellos. La función de demanda para los paneles se ha calculado como $p=-0.005 q+500$, en donde q es igual al número de unidades de demanda anual y $\mathrm{P}$ es igual al precio en dólares. La capacidad de producción anual del fabricante es de 20000 unidades. El costo total de producción de q paneles está adecuadamente representado mediante la función $C=150000+100 q+0.003 q^{2}$.

a) ¿Cuántas unidades q deben producirse para maximizar la utilidad anual?

b) ¿Qué precio debe cobrarse por cada panel?

c) Calcule la utilidad máxima.

Solución:

$$
\begin{aligned}
& U=I_{t}-C_{t} \\
& I_{t}=p \cdot q \\
& I_{t}=(-0.005 q+500) \cdot q \\
& I_{t}=-0.005 q^{2}+500 q \\
& U=-0.005 q^{2}+500 q-150000-100 q-0.003 q^{2}
\end{aligned}
$$

Función objetivo: $U(q)=-0.008 q^{2}+400 q-150000 \quad 0 \leq q \leq 20000$

$$
\begin{aligned}
& U^{\prime}(q)=-0.016 q+400 \quad U^{\prime}(q)=0 \\
& -0.016 q=-400 \\
& q=\frac{-400}{-0.016}=25000 \\
& U(0)=-150000 \\
& U(20000)=4650000 \quad \text { Utilidad máxima. }
\end{aligned}
$$

a) Para maximizar la utilidad anual deben producirse 20000 unidades.

b) $p=-0.005 \cdot(20000)+500=400$

En este caso no es necesario esclarecer el carácter de cada punto crítico. La situación es distinta cuando el intervalo no es cerrado, no es acotado o no es ni cerrado ni acotado. Por eso, no es aplicable el teorema de Weierstrass, ya que no garantiza la existencia del extremo global por lo que es necesario esclarecer el carácter de cada punto crítico y analizar 
el comportamiento de la función cuando la variable independiente tiende a cada uno de los extremos del intervalo.

6. El costo total de producción de q unidades de un cierto producto se describe mediante la función $C=100000+1500 q+0.2 q^{2}$ en donde $C$ es el costo total en dólares. Determine cuántas unidades q deben producirse con el fin de minimizar el costo promedio por unidad.

Solución:

$$
\begin{aligned}
& C=f(q)=\frac{100000}{q}+1500+0.2 q \quad f^{\prime}(q)=0 \\
& f^{\prime}(q)=\frac{-100000}{q^{2}}+0.2 \quad \frac{-100000}{q^{2}}+0.2=0 \\
& \frac{-100000}{q^{2}}=-0.2 \\
& \frac{-100000}{-0.2}=q^{2} \\
& q^{2}=500000 \\
& q=707.11 \\
& -\frac{+}{707.11}
\end{aligned}
$$

Existe un mínimo relativo para $\mathrm{f}(\mathrm{q})$ (costo promedio) cuando $\mathrm{q}=707.11$

7. La demanda por el producto de una compañía varía con el precio que la compañía cobra por el producto. La firma ha determinado que los ingresos anuales totales I (indicados en miles de dólares) son una función del precio $\mathrm{p}$ (indicado en dólares). Específicamente $I=f(p)=-50 p^{2}+500 p$

- Determinar el precio que debe cobrarse con el fin de maximizar los ingresos totales.

- ¿Cuál es el valor máximo de los ingresos anuales totales?

Solución:

a) $f^{\prime}(q)=-100 p+500$

$-100 p+500=0$

$$
p=5
$$

$f^{\prime \prime}(p)=-100<0 \quad \therefore \mathrm{p}=5$ es un máximo relativo sobre $\mathrm{f}(\mathrm{p})$ en $\mathrm{p}=5$ 
b) $\operatorname{Im} a ́ x=f(5)=-50 \cdot(5)^{2}+500 \cdot 5$

$$
\begin{aligned}
& =-1250+2500 \\
& =1250
\end{aligned}
$$

Los ingresos anuales totales son máximos en 1250 miles de dólares (1.25 millones) cuando la compañía cobra 5 dólares por unidad.

\section{Conclusiones}

1. La selección y aplicación de las herramientas Matemáticas en el proceso formativo del tecnólogo de la salud ha permitido revelar las relaciones esenciales que sustentan su representación teórica para lograr un proceso pedagógico eficiente y eficaz.

2. El proceso de formación técnico profesional del tecnólogo de la salud revela la lógica de la dinámica de este proceso a través de la especificidad y generalidad de los problemas profesionales que enfrenta el profesional.

3. El desarrollo del pensamiento técnico profesional facilita los procesos racionales para la resolución de problemas, donde su dinámica aún no satisface las demandas de salud que requiere la sociedad.

4. El conocimiento de la Matemática aplicada a la solución de los problemas profesionales requiere de operaciones mentales que le permitan al tecnólogo de la salud tener la capacidad de asimilar e interactuar con las nuevas tecnologías en su desempeño profesional.

5. La complejidad tecnológica a la que se enfrentan los tecnólogos de la salud durante el ejercicio de su profesión así como los complejos problemas profesionales que tendrán que solucionar hacen necesario el perfeccionamiento y desarrollo de su formación técnico profesional.

\section{Referencias}

Álvarez, Pedro, Corcho, Paula y Guerrero, María del Mar. (2001). Una forma eficiente de elaborar un programa de Matemáticas en LADE y LE. Las Palmas de Gran Canaria: Ed. ASEPUMA.

Chibas, Julio Daniel. (2010). La formación del pensamiento lógico de los estudiantes de la carrera licenciatura en Cultura Física de la Facultad de Santiago de Cuba. (Tesis doctoral). Universidad de Oriente, Cuba. 
Chirino Ramos, María Victoria. (2005). El trabajo independiente desde una concepción desarrolladora del proceso de enseñanza - aprendizaje. El trabajo independiente. Sus formas de realización. La Habana: Editorial Pueblo y Educación.

Fuentes González, Homero. (2009). La Concepción Científica Holística - Configuracional. Una alternativa en la construcción del conocimiento científico. Su aplicación en la formación de los profesionales de la Educación Superior en la contemporaneidad. (Tesis en opción al Grado Científico de Doctor en Ciencias). Centro de Estudios de Educación Superior "Manuel F. Gran". Universidad de Oriente. Santiago de Cuba. Cuba.

González de la Barrera, Lucas G., Mazarío Triana, Ana Cecilia y Mazarío Triana, Israel. (Marzo del 2000). Propuesta de un diseño de trabajo grupal para abordar problemas en las clases de ciencia. Ciudad de Matanzas: Editorial Universitaria del Ministerio de Educación Superior de la República de Cuba.

Guerrero Pupo, Julio C., Amell Muñoz, lleana, Cañedo Andalia, Rubén. (2004). Tecnología, tecnología médica y tecnología de la salud: algunas consideraciones básicas. Acimed; 12(4). Recuperado de http://bvs.sld.cu/revistas/aci/vol12 4 04/aci07404.htm

Labarrere Reyes, Guillermina y Valdivia Pairol, Gladys E. (1988). Pedagogía. La Habana: Editorial Pueblo y Educación.

Majmutov, Mirza I. (1983). La enseñanza problémica. La Habana: Editorial Pueblo y Educación.

Rico Montero, Pilar. (2002). Algunas características de la actividad de aprendizaje y de desarrollo de los alumnos. Compendio de Pedagogía. La Habana: Editorial Pueblo y Educación.

Santana de Armas, Hilario. (2003). Las formas de trabajo y de pensamiento matemático en la enseñanza de la Matemática [material digital]. La Habana, Cuba: Instituto Superior Pedagógico. "Enrique José Varona".

Santos Trigo, Luz Manuel. (1994). La Resolución de Problemas en el Aprendizaje de las Matemáticas. Departamento de Matemática Educativa. México: CINVESTAV-IPN.

Tarifa Lozano, Lourdes y González Romero, Rosa. (Marzo del 2000). Algunas flexiones sobre la resolución de problemas matemáticos. Ciudad de Matanzas: Editorial Universitaria del Ministerio de Educación Superior de la República de Cuba.

Torres Fernández, Paúl. (1997). La enseñanza problémica de la Matemática. Una concreción vigotskiana en la educación Matemática. Pedagogía' 97, Encuentro por la unidad de los educadores latinoamericanos, febrero, La Habana, Cuba. 